\title{
COMMENTARX
}

\section{The long-term health consequences of disasters and mass traumas}

\section{Sandro Galea}

$\infty \quad$ See related article page $\mathrm{I} 279$

I $\mathrm{n}$ recent years, high-profile disasters, including the September II, 200I terrorist attacks in New York City, the March II, 2004 train bombings in Madrid and Hurricane Katrina on the US Gulf Coast, have been accompanied by a surge in scientific interest about the physical and mental health consequences of disasters and mass traumas. Several studies have amply shown that these events are associated with substantial psychological illness among those who are directly involved in the event, among people in the general population, and among special groups, including rescue workers. ${ }^{1,2}$ There is also evidence, albeit less robust, that considerable physical illness occurs after these events, over and above initial trauma-related injury. ${ }^{3}$ In this issue of $C M A J$, Morren and colleagues ${ }^{4}$ add to this body of evidence by using a Dutch electronic medical record database that allows pre- to postdisaster health comparisons as well as comparisons between large case and control groups. The authors show that there is an increase in physical and psychological illness after a disaster in the short term, and that physical unwellness persists in the long term, even after psychological problems have subsided. Their analysis provides an opportunity for reflection about the long-term health consequences of disasters and mass traumas as well as their implications for population heath and clinical practice.

Box $\mathrm{I}^{1,2,5}$ provides a summary of the evidence on the consequences of disasters and mass traumas. Although we now have a large body of literature on the subject, it predominantly features cross-sectional or short-term studies. ${ }^{1}$ Evidence about the longer-term effects of these events is therefore important. Morren's group were able to document long-term effects of the Enschede disaster by means of the CAS system (the classification of complaints, diseases, and causes of ill health that is used by company and insurance doctors), a unique system of electronic medical records, and in doing so provide evidence about illness and disability even years after this disaster. Importantly, the subpopulation who constitute the subject of this study, rescue workers, are younger and, we would expect, healthier at baseline than the general population. Therefore, the observation that physical health in this group is a persistent concern long after a disaster highlights the substantial impact that mass traumas have on well-being, and thus the central role that these events may play in shaping population health.

Public health concern about disasters has long focused on preparedness exercises and the development of surge capacity in emergency departments. Although focus on an effective early response and on coping with the immediate consequences of mass traumas is clearly important, Morren and colleagues' report illustrates that thinking only about the acute population-health consequences of disasters is essentially considering only the tip of the iceberg. The evidence for

Box 1: What we know and don't know about the health consequences of disaster and mass trauma: a summary*

- The population burden of mental and physical pathology in the aftermath of disasters and mass traumas is substantial:

- Posttraumatic stress disorder, depression and generalized anxiety are the most common psychopathologic consequences

- Physical pathology after such events includes immediate event-related injury, nonspecific medical symptoms, and respiratory and cardiovascular disease

- Substance use increases after disasters $†$

- In general, pathologies are most prevalent (in descending order of burden) among:

1. People injured during the mass trauma

2. Rescuers

3. People who lost homes, property, belongings

4. The families of those injured

5. The general population

- Psychopathology and physical pathology co-occur after disasters and mass traumas:

- People with psychopathology are more likely to become physically ill

- People with physical illness or injury are more likely to experience psychological problems

- Most evidence is from cross-sectional studies or studies with short-term follow-up; we therefore have relatively little information about the trajectory of mental or physical health after a disaster

- The costs of disaster and mass trauma likely include:

- Short-term costs of treating physical injuries and illness and mental problems

- Social and economic costs, long after an event, of the persistent mental and physical health consequences and behaviour changes

*Much of the information in this summary is drawn from references 1,2 and 5: Psychiatry 2002;65:207-39 and Epidemiol Rev 2005;27:78-91 and 92-106. †The evidence supporting increased substance use is less robust than that for the other disaster effects shown. 
long-term physical health consequences of mass traumas among rescue workers and, as shown in other work, in the general population ${ }^{5}$ suggests that the consequences of mass trauma on burden of care may be profound. Persistent illness after an event likely will result in greater use of physician services, ${ }^{6}$ potentially including both scheduled and unscheduled physician visits, ${ }^{7}$ and changes in patterns of need for physician care that extend well beyond the immediate few days after a disaster.

Recognition that physical symptoms may persist for several years after exposure to a disaster, then, has implications for clinicians - perhaps particularly for primary care providers. Patients with physical symptoms, such as those studied here by Morren and colleagues, may not directly attribute their symptoms to their disaster experience. Given the comorbidity between physical and psychological symptoms, and the contribution of psychological pathology to the course of physical ill health, a high degree of suspicion on the part of clinicians may be essential to understand the causes of symptoms and to provide effective care. This issue may be less of a concern when dealing with special populations, such as rescue workers, for whom clinicians may have a higher degree of a priori suspicion about the role that a traumatic event may play in their health complaints. However, the findings of Morren's group, together with other work that has documented physical symptoms in other populations in the aftermath of disasters, ${ }^{5}$ serves as an important reminder that traumatic event experiences should be central to clinicians' diagnostic thinking when they treat patients who may have experienced mass traumas.

Intriguingly, Morren and colleagues show that physical symptoms persist in the long term, beyond psychological symptoms. There is little other empiric work that has considered the concurrent trajectory of both. However, previous studies have shown that a large proportion of early postdisaster psychological symptoms resolve in the first few months after a disaster, ${ }^{8}$ that substance use after mass trauma may remain elevated in the general population after psychological symptoms have decreased in prevalence, ${ }^{9}$ and that there is a relation between mental and physical consequences of traumas. ${ }^{7}$ Future work to explore the biologic processes that link trauma exposure and subsequent pathology may shed light on the interrelations between traumatic-event experiences and psychological and physical illness.

Morren and colleagues also found evidence for delayed increases in symptoms, years after the disaster event. Most studies of follow-up after trauma suggest that psychological symptoms start in the immediate aftermath of an exposure, ${ }^{10}$ which implies a monotonic trajectory of symptom resolution over time posttrauma. However, much of the published literature is based on studies that sampled only people directly af- fected by disasters. ${ }^{2}$ By sampling from a broader population, the study by Morren's group may be providing evidence of other patterns of progression of pathology. Consistent with this, a longitudinal study in Northern China found that the prevalence of psychopathology was higher at 9 months than at 3 months after an earthquake, ${ }^{11}$ and a study of firefighters exposed to an Australian bushfire found that the psychological symptoms fluctuated considerably over time. ${ }^{12}$

The paucity of existing evidence about the trajectory of physical symptoms, and our limited understanding of the mechanisms that link trauma with physical and psychological symptoms, mean that much more work needs to be done to consider systematically the determinants of different patterns of pathology in the aftermath of disasters and mass traumas. The work in this issue of CMAJ by Morren and colleagues is a step in this direction.

Sandro Galea is with the Departments of Epidemiology at the University of Michigan School of Public Health, Ann Arbor, Mich., and at Columbia University Mailman School of Public Health, New York, NY.

Competing interests: None declared.

Acknowledgements: Funded in part by grants from the National Institutes of Mental Health DAor 7642 and MHo78152.

\section{REFERENCES}

I. Norris FH, Friedman MJ, Watson PJ, et al. 60,00o disaster victims speak: part I. An empirical review of the empirical literature, I98I-200I. Psychiatry 2002;65:207-39.

2. Galea $S$, Nandi A, Vlahov D. The epidemiology of post-traumatic stress disorder after disasters. Epidemiol Rev 2005;27:78-9I.

3. Berrios-Torres SI, Greenko JA, Phillips M, et al. World Trade Center rescue worker injury and illness surveillance, New York, 200I. Am J Prev Med 2003;25:79-87.

4. Morren M, Dirkzwager AJE, Kessels FJM, et al. The influence of a disaster on the health of rescue workers: a longitudinal study. $C M A J$ 2007;176(9):1279-83.

5. Van den Berg B, Grievink L, Yzermans J, et al. Medically unexplained physical symptoms in the aftermath of disasters. Epidemiol Rev 2005;27:92-106.

6. Dorn T, Yzermans CJ, Kerssens JJ, et al. Disaster and subsequent healthcare utilization: a longitudinal study among victims, their family members, and control subjects. Med Care 2006;44:58I-9.

7. Fagan J, Galea S, Ahern J, et al. Relationship of self-reported asthma severity and urgent health care utilization to psychological sequelae of the September II, 200 I terrorist attacks on the World Trade Center among New York City area residents. Psychosom Med 2003;65:993-6.

8. Galea S, Vlahov D, Resnick H, et al. Trends of probable post-traumatic stress disorder in New York City after the September II terrorist attacks. Am J Epidemiol 2003;158:514-24.

9. Vlahov D, Galea S, Ahern J, et al. Sustained increased consumption of cigarettes, alcohol, and marijuana among Manhattan residents after September II, 200I. Am J Public Health 2004;94:253-4.

Io. North CS, Nixon SJ, Shariat S, et al. Psychiatric disorders among survivors of the Oklahoma city bombing. JAMA I999;282:755-62.

II. Wang X, Gao L, Shinfuku N, et al. Longitudinal study of earthquake-related PTSD in a randomly selected community sample in north China. Am J Psychiatry 2000; I57:1260-6.

I2. McFarlane AC, Papay P. Multiple diagnoses in posttraumatic stress disorder in the victims of a natural disaster. J Nerv Ment Dis I992;180:498-504.

Correspondence to: Dr. Sandro Galea, Department of

Epidemiology, University of Michigan School of Public Health, I2I4 S. University Ave., Ann Arbor MI 48104; sgalea@umich.edu 